\title{
Assessment of the Blood Parameters, Cardiac and Liver Enzymes in Oral Squamous Cell Carcinoma Following Treated with Injectable Doxorubicin-Loaded Nano-Particles
}

\author{
Monir Moradzadeh Khiavi', Enayat Anvari ${ }^{1}$, Hamed Hamishehkar ${ }^{3}$, \\ Khadijeh Abdal ${ }^{4 *}$
}

\begin{abstract}
Purpose: Oral squamous cell carcinoma (OSCC) is the most common and most malignant disorder of the oral cavity. Standard cancer treatments have many complications for patients. Nausea, vomiting, and perturbation in blood cells are the most common side effects when using Doxorubicin (Dox) for the treatment of OSCC. Use of Doxorubicin-loaded nano-particles (n-Dox) give rise to increase its biological efficacy and the rapeutic effects. This study assessed the efficacy of the injectable form of the n-Doxon blood parameters and cardiac and liver enzymes compared to the commercial form of Dox in OSCC-induced by 4NQO in rats. Methods: 4-nitroquinoline-1-oxideas was used as a solution in drinking water for inducing OSCC during 14 weeks in male Sprague-Dawley rats. Four groups of animals were categorized randomly: first (OSCC+Dox), second (OSCC + n-Dox), third (OSCC) and, last, healthy animals. Results: Using n-Dox had no harmful effect on the number of white and red blood cells. Thrombocytopenia and leukopenia in animals treated with n-Dox was less than the other groups. Hemoglobin and hematocrit in all treated groups did not differ and were similar to the healthy control. Hepatic and cardiac enzymes did not show any significant difference in any of the groups. Conclusion: The results of this research showed that significant decreases in haematological changes occurred, including leukopenia and anemia, in an animal model of OSCC induced by 4-NQO following use of n-Dox with compare to Dox. Use of n-Dox is better than of Dox for treatment of OSCC.
\end{abstract}

Keywords: Oral squamous cell carcinoma- 4-nitroquinoline-1-oxide- Doxorubicin- Rat- oral cancer

Asian Pac J Cancer Prev, 20 (7), 1973-1977

\section{Introduction}

Oral squamous cell carcinoma (OSCC) is the most common and most malignant disorder in the oral cavity. It accounts more than $90 \%$ of oral malignancies (Neville et al., 2016). OSCC is important because of the high incidence of side effects or complications during treatment and a high mortality rate. Only about half of patients with oral cancer survive more than 5 years (Petersen, 2008; da Silva et al., 2011). OSCC could result in changes in the patient's appearance and reduction in the ability to eat and quality of life (Joensuu et al., 2008). Standard cancer treatments include surgery, chemotherapy, and radiotherapy. However, these have many complications and problems for patients (da Silva et al., 2011; Johansson et al., 2006). Chemotherapy is used for advanced cancers and multiple metastases because it is the only means by which cancer cells can be destroyed throughout the body (Johansson et al., 2006). Doxorubicin is one of the most effective anti-cancer drugs. It is prescribed for the treatment of many cancers, usually in combination with other cancer drugs. It is most often used in acute leukemia and lymphoma, and organ cancers of the lung, breast, bladder, stomach, thyroid and reproductive system (Johansson et al., 2006; Nowak et al., 2004) and vomiting is the most common side effect from Doxorubicin in its injectable form. Doxorubicin also disrupts the production of red blood cells and can cause blood clotting disorders, anemia and leukopenia.Therefore, its effect on blood parameters should be closely monitored (Cortes et al., 2007). To solve this problem and reduce the side effects of Doxorubicin, much research has been done, to include encapsulating the drug within nanoparticles such as liposomes (Pramanik et al., 2012; Lebold et al., 2009; Wohlfarta et al., 2011). Laboratory (cell and animal) and clinical studies have been conducted on nanoparticles of Doxorubicin. The results of these studies showed anti-tumor effects, increases in patient survival, positive

${ }^{I}$ Department of Oral and Maxillofacial Pathology, Faculty of Dentistry, Tehran University of Medical Sciences, Tehran, ${ }^{2}$ Department of Physiology, Faculty of Medicine, ${ }^{4}$ Department of Oral and Maxillofacial Pathology, Faculty of Dentistry, Ilam University of Medical Sciences, Ilam, ${ }^{3}$ Department of Drug Applied Research Center, Tabriz University of Medical Sciences, Tabriz, Iran. *For Correspondence: dr.faribaabdal@yahoo.com 
results of treatment, and reduction of side effects ( Bootz et al., 2005 ; Ambruosi et al., 2006 ; Khiavi et al 2017; Khiavi et al., 2015 ).

The effects of this new form of drug on blood parameters and cardiac and liver enzymes have not been studied yet. This study assessed the efficacy of the injectable form of nanoparticles of Doxorubicin compared to the commercial form in cancer induced by 4-nitroquinoline-1-oxide (4-NQO) in rats. The main aim of this study is to outline the assay of the cardiac and liver enzymes with haematological parameters in a rat model of oral squamous cell carcinoma following treated with the nano-drug form of Doxorubicin.

\section{Materials and Methods}

\section{Animals}

Male Sprague-Dawley rats $(150 \pm 15 \mathrm{~g})$ were purchased. All animal experiments were approved by the Animal Ethics Committee (approval license5/4/10259) and kept in accordance with the Tabriz University of Medical Sciences guidelines. They were kept under standard conditions (temperature; $22^{\circ} \mathrm{C}-25^{\circ} \mathrm{C}$ ) and 12 hour light-dark cycles. The animals had free access to tap water and standard food. The animals were distributed randomly into four groups $(n=15$ rats) that included two rats in each cage.

\section{Preparation of $n-D o x$}

The preparation procedure of nano-particles of doxorubicin was fully described by Salehi et al., (2015). In brief, some nano-particles of Doxorubicin were ultrasonically dispersed in a methotrexate solution for 5 minutes. After 24 hours in dark conditions, DOX-HCl was added to the methotrexate-nanoparticles mixture and was stirred using ultrasonics (Sonics VibraCell, VCX 130 PB, Newton, CT) for 5 minutes. The solution was kept under magnetic mixing under dark conditions for 24 hours. After this, the fine precipitates were sedimented over 2 hours. Dox-methotrexate-loaded nano-particles were centrifuged (14,000 rpm /15 min). The nano-drug was diluted with normal saline solution in the appropriate concentration before administration to animals.

\section{Induction of OSCC}

Many studies have been conducted of 4-nitroquinoline1-oxide (4-NQO)-induced OSCC to assay various stages of oral carcinogenesis (Sohrabi et al., 2009). 4-NQO (Sigma) was used at a concentration of 30ppm during 14 weeks (PMID:16448841).

\section{Design of protocol}

Four categories of animals were randomly selected and each group consisted of 14 rats. 4-NQO was dissolved in the drinking water.

First group (OSCC+Dox) served as a carcinoma cancer model and received 4-NQO at a concentration of 30ppm over 14 weeks. They also intravenously (IV) received doxorubicin $(1.5 \mathrm{mg} / \mathrm{kg})$ once a day on days 2,5 and 8 of the study.

Second group (OSCC+n-Dox) served as a carcinoma cancer model and received 4-NQO at a concentration of 30ppm over14 weeks. They also intravenously (IV) received nano-particles of doxorubicin $(1.5 \mathrm{mg} / \mathrm{kg})$ once a day on days 2, 5 and 8 of the study.

Third group (OSCC) served as a carcinoma cancer model and received 4-NQO at a concentration of $30 \mathrm{ppm}$ over 14 weeks without any treatment.

Fourth group(healthy animals) received no carcinogen or treatment.

One-way ANOVA and Kruskal-Wallis test were used for the comparison of the differences between experimental and control animals. A difference in the p-value at the level of 0.05 or less was considered significant.

\section{Results}

Our microscopic findings showed 4-NQO induced typical cancerous and pathological changes in the tongue epithelium of rats in OSCC groups (Figure 1). Using nano-Doxorubicin had little impact on the number of white and red blood cells, while those rats that received the commercial form of Doxorubicin has led to a sharp decrease in white and red blood cells $(\mathrm{p}<0 / 001)$. Thrombocytopenia and leukopenia in animals treated with nano-medicine were less than in the commercial form. The

Table 1. Mean \pm SD of Hematological Parameters in Different Groups after 15 Weeks from the Initial Period of Experiments

\begin{tabular}{lcccc}
\hline Hematological parameters & \multicolumn{2}{c}{ Groups } & Healthy \\
& & OSCC & & Without intervention \\
& Dox & n-Dox & - & - \\
\hline Leukocyte count $\left(\times 10^{3} / \mathrm{mm}^{3}\right)$ & $4.8 \pm 1.8 \dagger$, & $9.3 \pm 2.6$ & $11 \pm 2.2$ & $10.1 \pm 2.1$ \\
RBC count $\left(\times 10^{6} / \mathrm{mm}^{3}\right)$ & $6.9 \pm 0.1 *$ & $7.4 \pm 0.5$ & $7.3 \pm 0.5$ & $7.6 \pm 0.6$ \\
$\mathrm{Hb}(\mathrm{mg} / \mathrm{dl})$ & $12.3 \pm 0.04$ & $12.9 \pm 0.8$ & $12.8 \pm 0.8$ & $13.3 \pm 0.8$ \\
Hct $(\%)$ & $34.2 \pm 0.3$ & $35.7 \pm 2$ & $35.4 \pm 2.2$ & $36.8 \pm 2$ \\
Plt count $\left(\times 10^{3} / \mathrm{mm}^{3}\right)$ & $791 \pm 51 \dagger$ & $1022 \pm 107$ & $889 \pm 193$ & $1010 \pm 89$ \\
Neutrophil $(\%)$ & $10.5 \pm 1.2 *$ & $20.3 \pm 1$ & $10.6 \pm 0.7$ & $62 \pm 8.1$ \\
\hline Data & & & \\
\hline
\end{tabular}

Data showed a significant difference in several haematological parameters between the Doxorubicin treated (Dox) animals compared to another groups (*represents a $\mathrm{p}$ value of $\mathrm{p}<0.05$ compared to nano Doxorubicin (n-Dox), oral squamous cell carcinoma (OSCC) and healthyanimals, £ represents a $\mathrm{p}$ value of $\mathrm{p}<0.01$ compared to OSCC $+\mathrm{n}$-Dox and healthy animals, $\uparrow$ represents a $\mathrm{p}$ value of $\mathrm{p}<0.001$ compared to just only OSCC animals. RBC, red blood cells; Hb, hemoglubin; Hct, hematocrit; Plt, Platlet; $n$, Table 1. Mean \pm SD of hematological parameters in different groups after 15 weeks from the initial period of experiments. 15 animals in each group). 
Table 2. Mean \pm SD of Hepatic and cardiac enzymes in different groups after 15 weeks from the initial period of experiments

\begin{tabular}{lcccc}
\hline \multicolumn{3}{c}{ parameters } & \multicolumn{3}{c}{ Groups } \\
& \multicolumn{3}{c}{ Intervention } & Healthy \\
& Dox & n-Dox & - & - \\
\hline AST (IU/L) & $170 \pm 33$ & $144 \pm 26$ & $196 \pm 51$ & $113 \pm 31$ \\
ALT (IU/L) & $49 \pm 13$ & $43 \pm 11$ & $39 \pm 7$ & $37 \pm 11$ \\
LDH (IU/L) & $400 \pm 66$ & $337 \pm 49$ & $310 \pm 81$ & $327 \pm 43$ \\
ALP (IU/L) & $140 \pm 38$ & $112 \pm 17$ & $98 \pm 26$ & $110 \pm 29$ \\
CK (IU/L) & $497 \pm 118$ & $383 \pm 126$ & $279 \pm 88$ & $410 \pm 117$ \\
\hline IU/L, AST (SGOT), ALT (SGPT), ALP, LDH and CK represent \\
the international unit per liter, aspartate aminotransferase, alanine \\
aminotransferase, alkaline phosphatase, Lactate dehydrogenase and \\
Creatine kinase respectively.
\end{tabular}

results show that hemoglobin and hematocrit levels in all treated groups did not differ and were similar to that of the healthy control (Table 1).

Hepatic (SGOT, SGPT) and cardiac (LDH,ALK, CK) enzymes were tested for significant differences between treatment groups and control group but did not show any significant differences $(\mathrm{P}>0 / 12)$ (Table 2).

\section{Discussion}

The influences of n-Dox, an anti-neoplastics drug, were evaluated in a 4-NQO-induced OSCC animal model outcome. Accordingly, liver and cardiac enzymes and haematological changes were considered for this study. Our results indicated that use of n-Dox has fewer side effects than Dox.

Currently chemotherapy is used for approximately $50 \%$ of human cancers. Nevertheless, the main problem related to chemotherapy is the inability to deliver a suitable dose of pharmaceuticals to target cancerous tissue without inducing toxicity in normal tissues (Qin et al., 2017). Here, we focused on use of n-Dox in treating OSCC. Nevertheless, it was necessary to create appropriate pharmaceutical formulations to deliver effective doses of drug to the site of action and reduce the drug side effects. Polymer-based nanoparticles and lipids with a heat-sensitive polymer coating were designed as an anti-cancer drug delivery system. This system is loaded with Dox and is sensitive to temperature and $\mathrm{pH}$. Drug loaded nanoparticles provide targeted release within tumor tissue that has a lower $\mathrm{pH}$ (slightly acidic) and a higher temperature than normal physiological condition (Wei et al., 2015). The polymer carrier maintains drug stability and prevents release in the normal $\mathrm{pH}$ and temperature environments. However, in cancerous tissue, the polymer loses its structure and releases the drug due to changes in $\mathrm{pH}$ and temperature. Accordingly, it can be assumed that the drug will be directed specifically to the target tissue (tumor tissue) and side effects in other tissues will be reduced (Wei et al., 2015; Wang et al., 2010). Dox embedded with DNA gives rise to activation of various molecular signals in the AMPK pathway and influences the $\mathrm{Bcl}-2 / \mathrm{Bax}$ apoptosis pathway which induces apoptosis.

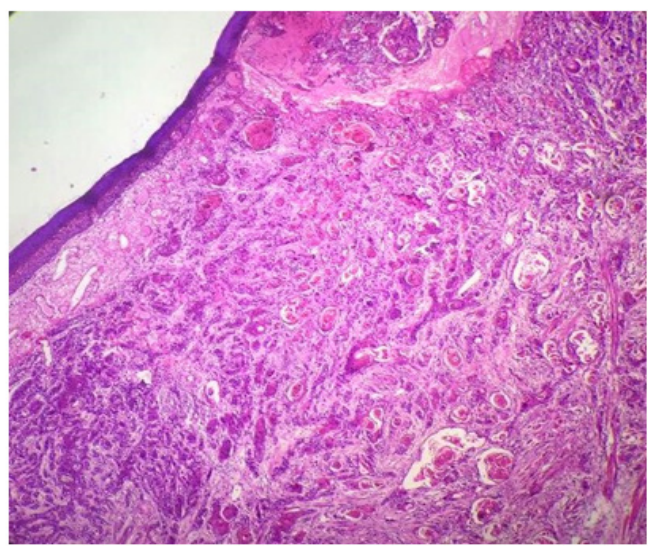

Figure 1. Well Differentiated Squamous Cell Carcinoma, Dysplastic Epithelial Cells are invading the lamina propria with keratin pearl formation $(\mathrm{H}$ and $\mathrm{E}, \times 200$, scale bar $=100 \mu \mathrm{m}$ )

It suppresses nucleic acid synthesis and excites apoptosis through promotion of $\mathrm{p} 53$ phosphorylation at Ser15 and acetylaion at Lys373/382, similar to n-Dox (Abbasi et al., 2014). This means that the Dox effect is mediated on Bcl2 expression through the p53 route. Both Dox and n-Dox can induce apoptosis by affecting the p 53 gene expression within tumor all cells and influence the other genes and pathways to fight cancerous cells (Abbasi et al., 2014; Supriya et al., 2016 ). N-Dox is used for the treatment of ovarian cancer, metastatic breast cancer, and Kaposi's sarcoma associated with AIDS (Laginha et al., 2013). Using n-Dox could decrease the histo-pathological grade, cellular proliferation, and mortality rate and weight gain compared to Dox in an animal model of OSCC (Khiavi et al., 2017; Khiavi et al., 2015). It is said that the n-Dox is more effective and this was confirmed by the results of our research (Laginha et al., 2013 ). In a study carried out by Bootz 2004, n-Dox utilization had fewer side effects and more anti-tumor effect than Dox (Ambruosi et al., 2006; Bootz et al., 2004). In a study conducted by Lingha on patients with breast cancer, use of n-Dox had more biological efficacy (Laginha et al., 2013). Studies by Petri et al., (2007) and Steiniger et al., (2004) on patients with brain tumors showed that n-Dox had higher therapeutic effects than its commercial form. The effectiveness, low side effects and especially reduced toxicity are the principal advantages compared with the free form of Dox and this thus increases the efficacy of treatment (Bootz et al., 2005; Laginha et al., 2013).

According to a study done by Wohlfart in which n-Dox was shown to be much more efficient than Dox, it was therefore suggested to use nanoparticles for human glioblastomas as a non-invasive therapy (Wohlfarta et al., 2011). Other researches carried out by Stefanie and Meang and Liming demonstrated the efficacy of n-Dox for a formulation of anti-cancer drugs to improve cancer therapy (Stefanie et al., 2011; Maeng et al., 2010; Liming et al., 2010). Here, the second group was considered in evaluating possible side effects of n-Dox. To assess the toxic effect as in vivo of the n-Dox, several biochemical indices were measured, such as aspartate aminotransferase (AST), alanine transaminase (ALT), lactate dehydrogenase $(\mathrm{LDH})$, and creatine kinase MB (CK-MB). These 
parameters did not show significant differences among all of the groups, which indicates that the toxic effects of n-Dox on liver and heart are similar to Dox. Since n-Dox treated group did not show significant changes in blood parameters compared to the Dox treated group, it can be concluded that $n$-Dox is more effective than Dox. Thus, our results confirm results of the previous studies on the effectiveness of n-Dox. Drugs loaded with nanoparticles are more efficient in the treatment of various cancers. However, more investigations are needed for other possible complications.

In conclusion the results of this research showed that significant decreases in haematological changes occurred, including leukopenia and anemia,in an animal model of OSCC induced by 4-NQO following use of n-Dox with compare to Dox. Use of n-Dox is better than of Dox for treatment of OSCC .However, further experiments are necessary to clarify the advantages and disadvantages of usingn-Dox for the treatment of human cancers.

\section{Acknowledgements}

This is a self financed study and we have received no financial support from elsewhere. However we'd like to acknowledge Drug Applied Research Center of Tabriz University of Medical Sciences-Iran and also the Research Center of Tabriz International Hospital for the valuable technical support.

\section{Ethical Issues}

All the ethical and the humanity considerations were performed according to the Helsinki humanity research declaration during the experiments and the euthanasia of the animals. All the animals' experiments were approved by the Ethics Committee of the Tabriz University of Medical Sciences.

\section{Conflict of Interest}

The authors report no conflicts of interest.

\section{References}

Abbasi MM, Monfaredan JE, Seidi A, et al (2014). Oral and IV dosages of Doxorubicin-Methotrexa loaded nanoparticles inhibit progression of oral cancer by down regulation of matrix Methaloproteinase 2 expression in vivo. Asian Pac J Cancer Prev, 15, 10705-1.

Ambruosi A, Gelperina S, Khalansky A, et al (2006). Antitumor effect of doxorubicin loaded in poly(butyl cyanoacrylate) nanoparticles in rat glioma model, influence of formulation parameter. J Microencapsul, 23, 582-92.

Bootz A, Russ T, Gores F, et al (2005). Molecular weights of poly(butyl cyanoacrylate) nanoparticles determined by mass spectrometry and size exclusion chromatography. Eur $J$ Pharm Biopharm, 60, 391-9.

Bootz A, Vogel V, Schubert D, et al (2004). Comparison of scanning electron microscopy, dynamic light scattering and analytical ultracentrifugation for the sizing of poly (butyl cyanoacrylate) nanoparticles. Eur J Pharm Biopharm, 57, 369-75.

Cortés-Funes H, Coronado C (2007). Role of anthracyclines in the era of targeted therap. Cardiovasc Toxicol, 7, 56-60.

da Silva SD, Ferlito A, Takes RP, et al (2011). Advances and applications of oral cancer basic research. Oral Oncol, 47, 783-91.

Joensuu H (2008). Systemic chemotherapy for cancer: from weapon to treatment. Lancet Oncol, 9, 304.

Johansson S, Goldenberg D, Griffiths G, et al (2006). Elimination of HIV-1 infection by treatment with a doxorubicinconjugated anti-envelope antibody. AIDS, 20, 1911-15.

Khiavi MM, Abdal K, Abbasi MM, et al (2017). Comparison of injectable doxorubicin and its nanodrug complex chemotherapy for the treatment of 4-nitroquinoline-1-oxide induced oral squamous cell carcinoma in rats. Indian J Med Res, 145, 112-7.

Khiavi MM, Rostami A, Hamishekar H, et al (2015). Therapeutic effcacy of orally delivered Doxorubicin Nanoparticles in Rat tongue cancer induced by 4-Nitroquinoline 1-Oxide. Adv Pharm Bull, 5, 209-16.

Laginha KM, Verwoert S, Charrois GJ, et al (2005). Determination of doxorubicin levels in whole tumor and tumor nuclei in murine breast cancer tumors. Clin Cancer Res, 1, 6944-9.

Lebold T, Jung C, Michaelis J, et al (2009). Nanostructured Silica materials as drug-delivery systems for Doxorubicin: single molecule and cellular studies. Nano Lett, 9, 2877-83.

Liming Z, Qinghuan Z, Liwei L, et al (2010). Functional Graphene Oxide as a nanocarrier for controlled loading and targeted delivery of mixed anticancer drugs. Small, 6, 537-44.

Maeng JH, Jung KH, Bae YH, et al (2010). Multifunctional doxorubicin loaded superparamagnetic iron oxide nanoparticles for chemotherapy and magnetic resonance imaging in liver cancer. Biomaterials, 31, 4995-5006.

Neville BW, Damm D, Allen CM, et al (2016). Oral and maxillofacial pathology. Epithelial Pathology. Elsevier, St. Louis, Missouri pp 1 number 374.

Nowak AK, Chow PK, Findlay M (2004).Systemic therapy for advanced hepatocellular carcinoma: a review. Eur J Cancer, 40, 1474-84.

Petersen PE (2014). Oral cancer prevention and control - The approach of the World Health Organization. Oral Oncol, 45, 454-60.

Petri B, Bootz A, Khalansky A, Muller R, et al (2007). Chemotherapy of brain tumour using doxorubicin bound to surfactant-coated poly(butyl cyanoacrylate) nanoparticles: revisiting the role of surfactants. J Control Release, 117, $51-8$.

Pramanik D, Campbell NR, Das S, et al (2012). A composite polymer nanoparticle overcomes multidrug resistance and ameliorates doxorubicin-associated cardiomyopathy. Oncotarget, 3, 640-50.

Qin W, Huang J, Chen Z, et al (2017). Nanomaterials in targeting cancer stem cells for cancer therapy. Front Pharmacol, 8,1 .

Salehi R, Hamishehkar H (2015). Smart thermo/pH responsive magnetic nanogels for the simultaneous delivery of doxorubicin and methotrexate. Int J Pharm, 487, 274-84.

Sohrabi M, Soleimani J, Roshangar L, et al (2009). The effect of dietary and topical celecoxib on 4-Nitroquinoline-1oxide-induced lingual epithelium alterations in rat. $J$ Pak Med Assoc, 11, 769-74.

Stefanie W, Svetlana G, Olga M, et al (2011). Efficient chemotherapy of Rat glioblastoma using doxorubicin-loaded PLGA nanoparticles with different stabilizers. PLoS One, 6, e19121.

Steiniger S, Kreuter J, Khalansky AS, et al (2004). Chemotherapy of glioblastoma in rats using doxorubicin-loaded nanoparticles. Int J Cancer, 109, 759-67.

Supriya R, Tam BT, Pei XM, et al (2016). Doxorubicin induces inflammatory modulation and metabolic dysregulation in diabetic skeletal muscle. Front Physiol, 27, 323. 
Wang Y, Wei X, Zhang C, et al (2010). Nanoparticle delivery strategies to target doxorubicin to tumor cells and reduce side effects. Ther Deliv, 1, 273-87.

Wei T, Chen C, Lui J, et al (2015). Anticancer drug nanomicelles formed by self-assembling amphiphilic dendrimer to combat cancer drug resistance. Proc Natl Acad Sci U S A, 112, 2978-83.

Wohlfart S, Khalansky As, Bernreuther C, et al (2011). Treatment ofglioblastoma with poly(isohexyl cyanoacrylate) nanoparticles. Int J Pharm, 415, 244-51.

\section{(ब) $(\mathbb{8}$}

This work is licensed under a Creative Commons AttributionNon Commercial 4.0 International License. 